\title{
Lipid Profile of High Tetraplegia (Above C4) in Japan
}

\author{
Masaaki Okada*
}

Department of Rehabilitation Medicine, National Rehabilitation Center for Persons with Disabilities, 4-1 Namiki
Tokorozawa Saitama 359-8555, Japan

\begin{abstract}
Abstaract: Objective: To investigate whether the risk profile for coronary heart disease (CHD) is more favorable in individuals with high tetraplegia (neurological lesion above $\mathrm{C} 4$ ) than in healthy controls. (Because inactivity has a negative effect on the lipid profile.) Design: Case-control study. Setting: This research was performed in the rehabilitation hospital for the spinal cord injured persons. Participants: 19 healthy male controls (age-, height-, and body weight-matched) and 62 individuals with high tetraplegia (mean age $45.0 \pm 17.35$ years) with a mean time since injury of $16.9 \pm 23.88$ months were assessed the information was obtained from the 22 tetraplegics who had returned home from our hospital (all the other 40 left to other hospital), and recorded data on lipid values for comparison. Interventions: Not applicable. Main outcome measures: Total plasma cholesterol (TC), high-density lipoprotein cholesterol (HDL-C) and triglyceride (TG) concentrations were measured, and calculated the low-density lipoprotein cholesterol (LDL-C) concentration and the ratios of TC/HDL-C and LDL-C/HDL-C were calculated. Results: Significantly lower concentrations of TC, HDL-C and LDL-C were found in individuals with a SCI. However, no statistically significant difference was found between TC/HDL-C and LDL-C/HDL-C ratios of tetraplegic individuals and healthy controls. In individuals with high tetraplegia who were discharged home, HDL-C was still lower than normal control. Conclusions: Patients with high tetraplegia showed low HDL-C, but the TC/HDL-C and LDL-C/HDL-C ratios were normal.
\end{abstract}

Keywords: High tetraplegia, high-density lipoprotein cholesterol (HDL-C), lifestyle-induced disease.

\section{INTRODUCTION}

Today, persons with a spinal cord injury (SCI) live longer than in the past because of the improved therapeutic strategies for treating life-threatening complications, such as urinary tract infections and pressure sores, in these individuals. Therefore, management of these patients to avoid coronary heart disease (CHD) has become more important [1]. Previous studies have reported that persons with a SCI have lower concentrations of high-density lipoprotein cholesterol (HDL-C) than able-bodied persons. Subjects with a lesion level above $\mathrm{C} 4$ are included in some lipid studies. However, the data of these subjects were not analyzed separately for this group. The data belonged to the overall group of subjects with tetraplegia [2-5].

Doctors or nursing care managers who examine these persons with SCI must be aware of the importance of the prevention of lifestyle-induced disease, as one of the main causes of death in SCI is CHD [6]. It is thought that in individuals with high tetraplegia, HDL-C concentrations are lower than in tetraplegia below C5 or paraplegia because of the exercise limitation in high tetraplegics, due to the paresis itself. El-Sayed MS et al. [7] reported the effect of activity on HDL-C profile in persons with a SCI.

The objective of this study was to determine the serum lipid profile in individuals with high tetraplegia, and compare the result with the standard for the diagnosis of hyperlipidemia in Japan.

*Address correspondence to this author at the Department of Rehabilitation Medicine, National Rehabilitation Center for Persons with Disabilities, 4-1 Namiki Tokorozawa Saitama 359-8555, Japan;

E-mail: qgb02435@yahoo.co.jp

\section{METHODS}

Sixty-two male individuals were assessed with tetraplegia, admitted from January 1997 to December 2004, during their rehabilitation period in our center. All the patients had been bedridden until admission. The time since injury was $16.9 \pm 23.88$ months (range 2-132 months; Though the time since injury varies greatly, all of them had been admitted for rehabilitation for the $1^{\text {st }}$ time). The mean age of the patients was $45.0 \pm 17.35$ years (range 17-80 years). Their mean BMI was $18.95 \pm 3.62 \mathrm{~kg} / \mathrm{m} 2$. All were classified as having high tetraplegia (above C4), and the ASIA impairment scale indicated $41(66 \%)$ complete (ASIA class A) and $21(34 \%)$ incomplete (ASIA class B) injuries. Therefore all the patients had a motor complete lesion. Traffic accidents were the most common cause $(40 \%)$ of SCI, followed by falls from a height $16(26 \%)$, sports accidents $11(18 \%)$ and falls $7(11 \%)$, with three unknown $(4 \%)$. None of the individual with tetraplegia had anemia or low albuminemia. Only 3 had diabetes mellitus (treated with insulin), 26 were smokers before, but not after their SCI. No one took medications that could affect their cholesterol levels.

Body length was measured to the nearest centimeter in a supine position and body weight was measured on a balance beam scale designed to accommodate wheelchairs.

Total cholesterol (TC), triglyceride (TG), and HDL-C concentrations were measured using enzymatic tests on the Hitachi 7450 automated analyzer (Japan). And glucose concentrations were measured. All participants were measured within 3 days from admission. Low density lipoproteincholesterol (LDL-C) in $\mathrm{mg} / \mathrm{dl}$ was calculated according to the Friedewald equation [8] as follows: LDL-C $=$ TC-(HDL$\mathrm{C}+\mathrm{TG} / 5$ ). 
Among the 62 high tetraplegics, the relationship was investigated between body weight and serum cholesterol profiles in 22 patients who were discharged at home (the remaining 40 went to other hosp.), and obtained responses from $12(55 \%)$. Individuals who went to home were supposed to have a changed lipid profile due to their meal. The average age of these 12 respondents was $31.3 \pm 14.13$ years (range 17-58 years).

Data used was taken from physical examinations of 19 male staffs in our center in November 2005 as a control group. The control group with age-, height-, and body weight-matched to the subjects with a SCI. Their age was 49.2(SD 11.6) years, height was $1.69(0.063)$ meters, and body weight was $56.4(5.51) \mathrm{kg}$, mean BMI was 23.9(2.87) $\mathrm{kg} / \mathrm{m}^{2}$.

\section{Statistics}

A Welch's t-test for independent samples was applied to detect differences between groups for subject characteristics and serum lipid profiles. Results were considered significant at $\mathrm{p} \leqq 0.05$.

\section{RESULTS}

The results of the comparison of demographic parameters in SCI patients and controls are shown in Table 1. TC, HDL-C, and LDL-C were significantly lower in those with SCIs. TC/HDL-C and LDL-C/HDL-C ratios were not significantly different between the two groups.
Table 1. Comparing between whole-SCIs and Healthy Control

\begin{tabular}{|c|c|c|c|}
\hline & $\begin{array}{c}\text { whole SCIs } \\
(\mathbf{n = 6 2}) \\
\text { Mean(SD) }\end{array}$ & $\begin{array}{c}\text { healthy control } \\
(\mathbf{n = 1 9 )} \\
\text { Mean(SD) }\end{array}$ & p-value \\
\hline \hline age(year) & $45.0(17.35)$ & $49.2(11.6)$ & N.S. \\
\hline height(m) & $1.69(0.077)$ & $1.69(0.063)$ & N.S. \\
\hline weight $(\mathrm{kg})$ & $54.4(11.50)$ & $56.4(5.51)$ & N.S. \\
\hline BMI(kg/m2) & $18.95(3.62)$ & $19.86(2.68)$ & N.S. \\
\hline TC(mg/dl) & $165.23(33.09)$ & $204.79(35.70)$ & 0.00019 \\
\hline HDL-C(mg/dl) & $39.31(10.49)$ & $52.84(13.54)$ & 0.0006 \\
\hline TG(mg/dl) & $105.42(48.11)$ & $129(59.05)$ & N.S. \\
\hline LDL-C(mg/dl) & $105.20(28.15)$ & $126.15(38.58)$ & 0.043 \\
\hline Glucose(mg/dl) & $92.96(25.05)$ & $87.32(13.96)$ & N.S. \\
\hline TC/HDL-C & $4.48(1.35)$ & $4.14(1.50)$ & N.S. \\
\hline LDL-C/HDL-C & $2.87(1.08)$ & $2.60(1.38)$ & N.S. \\
\hline
\end{tabular}

\section{Age-Related Factors}

In Japan, spinal cord injuries are most prevalent among two age groups, those aged between 20 and 30 years (the 'younger' group) and those in their 60s (the 'older' group) [9], with this age distribution also true in our sample (Fig. 1).

The participants were divided into SCI-younger (under 45 years: the subjects' mean age was 45 years) and SCI-older

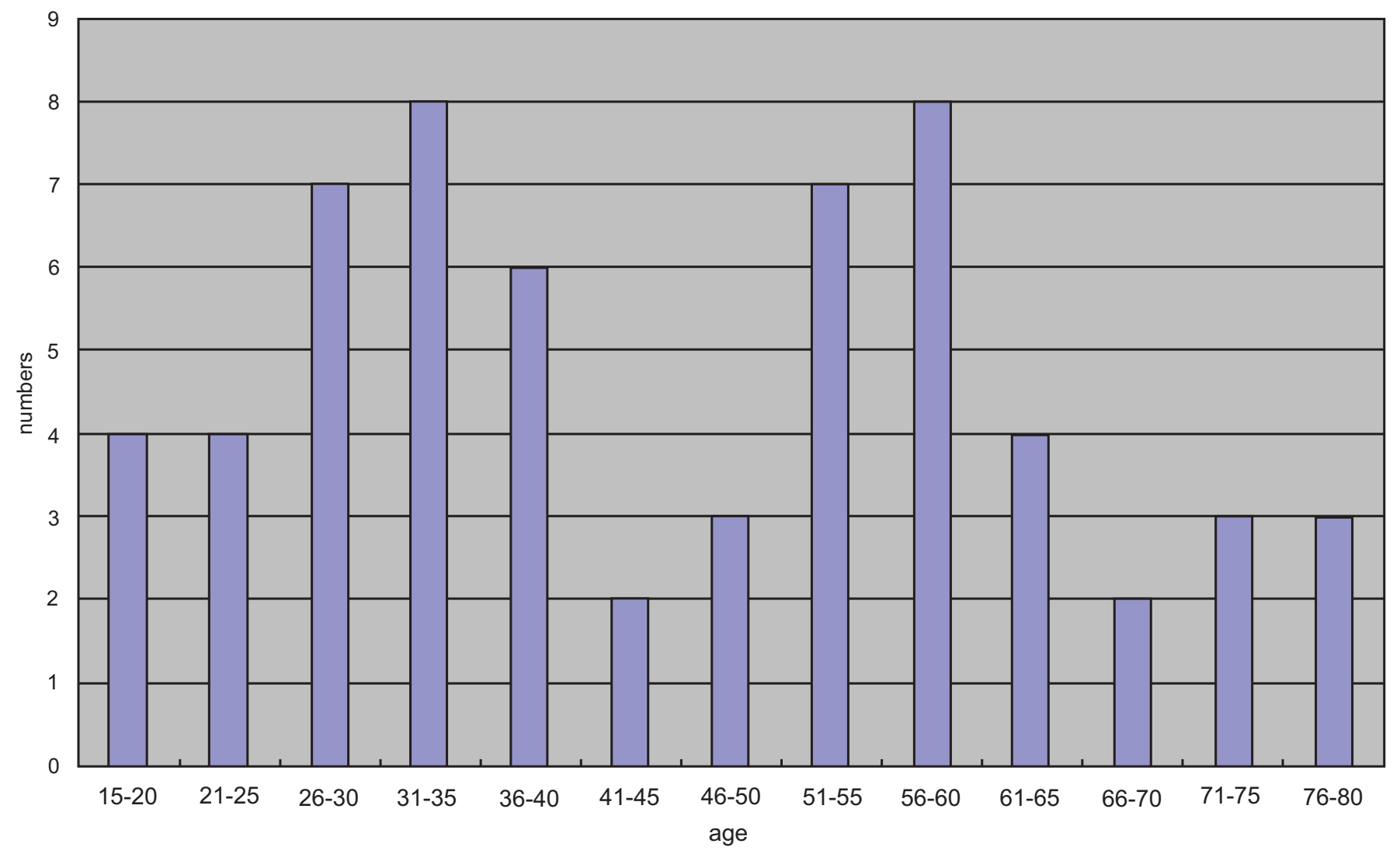

Fig. (1). Age distribution of tetraplegics. 
(over 46 years), they were then compared with two corresponding control groups: control-younger (20-30 years) and control-older (60 years), with the results of this comparison shown in Tables $\mathbf{2}$ and $\mathbf{3}$.

Table 2. Comparing between SCI-Younger and ControlYounger

\begin{tabular}{|c|c|c|c|}
\hline & $\begin{array}{c}\text { SCI-younger } \\
(\mathbf{n}=31) \\
\text { Mean(SD) }\end{array}$ & $\begin{array}{c}\text { control-younger } \\
(\mathbf{n}=7) \\
\text { Mean(SD) }\end{array}$ & p-value \\
\hline \hline age(year) & $29.84(7.15)$ & $34.71(1.70)$ & N.S. \\
\hline height(m) & $1.73(0.057)$ & $1.73(0.055)$ & N.S. \\
\hline weight(kg) & $55.13(13.54)$ & $52.81(4.88)$ & N.S. \\
\hline BMI $(\mathrm{kg} / \mathrm{m} 2)$ & $18.33(3.92)$ & $17.48(1.63)$ & N.S. \\
\hline TC(mg/dl) & $157.06(31.18)$ & $218.71(40.37)$ & 0.0053 \\
\hline HDL-C(mg/dl) & $38.12(11.24)$ & $51.43(13.69)$ & 0.046 \\
\hline TG(mg/dl) & $103.87(52.02)$ & $116.29(55.08)$ & N.S. \\
\hline LDL-C(mg/dl) & $95.53(32.25)$ & $144.03(42.09)$ & 0.023 \\
\hline Glucose(mg/dl) & $87.79(18.52)$ & $87.29(14.36)$ & N.S. \\
\hline TC/HDL-C & $4.37(1.49)$ & $4.65(2.21)$ & N.S. \\
\hline LDL-C/HDL-C & $2.74(1.18)$ & $3.12(1.93)$ & N.S. \\
\hline
\end{tabular}

Table 3. Comparing between SCI-Older and Control-Older

\begin{tabular}{|c|c|c|c|}
\hline & $\begin{array}{c}\text { SCI-older } \\
(\mathbf{n}=\mathbf{3 1}) \\
\text { Mean(SD) }\end{array}$ & $\begin{array}{c}\text { control-older } \\
(\mathbf{n = 1 2}) \\
\text { Mean(SD) }\end{array}$ & p-value \\
\hline \hline age(year) & $60.23(9.17)$ & $57.7(2.74)$ & N.S. \\
\hline height(m) & $1.66(0.077)$ & $1.67(0.057)$ & N.S. \\
\hline weight $(\mathrm{kg})$ & $53.63(9.17)$ & $58.4(4.89)$ & N.S. \\
\hline BMI $(\mathrm{kg} / \mathrm{m} 2)$ & $19.56(3.24)$ & $21.25(2.14)$ & N.S. \\
\hline TC(mg/dl) & $173.39(33.41)$ & $196.67(31.64)$ & 0.045 \\
\hline HDL-C(mg/dl) & $40.12(10.10)$ & $53.67(13.99)$ & 0.0081 \\
\hline TG(mg/dl) & $106.97(44.67)$ & $136.41(62.35)$ & N.S. \\
\hline LDL-C(mg/dl) & $111.77(23.43)$ & $115.71(33.89)$ & N.S. \\
\hline Glucose(mg/dl) & $98.52(29.93)$ & $87.33(14.37)$ & N.S. \\
\hline TC/HDL-C & $4.55(1.28)$ & $3.85(1.03)$ & N.S. \\
\hline LDL-C/HDL-C & $2.96(1.02)$ & $2.29(0.90)$ & N.S. \\
\hline
\end{tabular}

It was observed that TC, HDL-C, and LDL-C were significantly lower in the SCI-younger group than in the control-younger group.

Comparing the SCI-older and the control-older groups, it was found that TC and HDL-C were significantly lower in the SCI-older group than in the control-older group.

TC, TG, HDL-C, and LDL-C of individuals with tetraplegia living at home after being discharged from the hospital were not significantly different to those individuals that were compared with all the SCI individuals (TC 173.6(12.3) mg/dl, TG 104.4(55.9) mg/dl, HDL-C 41.0(14.1) mg/dl, LDL-C 111.4(33.8) mg/dl).
In 2002, the Japan Atherosclerosis Society issued 'Guidelines for Diagnosis and Treatment of Atherosclerotic Cardiovascular Diseases' (Table 4) [10], which proposed diagnostic reference values for serum lipids.

Table 4. The Guideline to Treat the Disease of Atherosclerosis (Japan Atherosclerosis Society)

\begin{tabular}{|l|l|l|}
\hline hypercholesterolemia & TC & $\geqq 220 \mathrm{mg} / \mathrm{dl}$ \\
\hline hyper low density lipoprotein-cholesterolemia & LDL-C & $\geqq 140 \mathrm{mg} / \mathrm{dl}$ \\
\hline lower high density lipoprotein-cholesterolemia & HDL-C & $<40 \mathrm{mg} / \mathrm{dl}$ \\
\hline hypertriglyceridiemia & & \\
\hline
\end{tabular}

In the healthy controls in this study, average values for serum lipids did not fall beneath the normal limit proposed in the above guideline, but the average value of HDL-C over the whole group of persons with a SCI, as well as in the SCIyounger group, was less than this standard. In the SCI-older group, the HDL-C concentration was $40.12 \mathrm{mg} / \mathrm{dl}$, which is very close to the limit of the normal value.

The number of persons agreeing with the guideline as a risk for atherosclerosis were 5 of SCIs in high TC (6 of control), 2 of SCIs in high LDL-C (4 of control), 22 of SCIs in low HDL-C (1 of control), and 11 of SCIs in high TG (7 of control).

\section{DISCUSSION}

In this study, the lipid profile of individuals with high tetraplegia was shown that TC, HDL-C, and LDL-C were lower than the healthy control. And, comparing to the 'Guidelines for Diagnosis and Treatment of Atherosclerotic Cardiovascular Diseases', TC and LDL-C were favorable, but HDL-C was unfavorable.

This tendency toward lower-than-normal levels of HDL-C is consistent with results of previous studies [11,12]. However, all such previous studies have involved individuals with paraplegia or quadriplegia (spinal injuries at or below the C5 level) who were able to operate a wheelchair without using their arms. This study is the first to focus on the serum lipid profile of high tetraplegia (above $\mathrm{C} 4$ ).

It was considerd that the cause of a low HDL-C concentration with other lipid values within normal limits in individuals with tetraplegia, regardless of age, is not the limited amount of exercise that these individuals can perform due to their paresis [13]. Since HDL-C levels of the individuals with tetraplegia had similar levels as individuals with lower levels of a SCI. The factor contributing to this lipid profile may be the controlled hospital diet provided in the hospital prior to arrival at the medical center.

The main reason why the age distribution of persons with a SCI has two peaks in Japan, different from that in Europe and America where there is only one peak in younger patients, has been reported to be that young patients get injured in traffic 
accidents, whereas older patients experience SCIs after incidents such as falls due to pre-existing cervical spondylosis. One cause of such pre-existing cervical spondylosis deformans is ossification of the posterior longitudinal ligament (OPLL). OPLL is thought to be correlated with the yellow races and is suggested to be associated with diabetes mellitus [14], but in this study, significantly no higher blood glucose levels were found either in the SCI-younger or SCI-older group than in the control group.

The TC/HDL-C and LDL-C/HDL-C ratios, strong predictors of CHD risk [15], did not differ significantly between the SCI group and the healthy control group. Though the time since injury was very broad in the subject group, there was no increased risk of CHD in the SCI group.

In this study, the low value of HDL-C persisted in the individuals who were discharged home from hospital. Forty patients were not followed up because they did not go home after being discharged from the hospital but were transferred to other medical institutes. This was because patients visited my hospital from many areas of the country and were then transferred to a variety of areas. Therefore, No data was available to compare lipid values between patients who went home after being discharged from the previous hospital and those who were transferred to other institutes.

Apstein et al. [16] examined the changes in serum lipid concentrations of patients with SCI following accidents. They described that HDL-C is markedly depressed following acute SCI and gradually returns toward normal by 1 year, and postulated that $44 \%$ of the increase in HDL$\mathrm{C}$ is due to an increase in physical activity and $56 \%$ to the SCI itself.

However, in this study, HDL-C values did not increase in any of the participants after they returned home following discharge from hospital, even when they were studied individually. Further study would be necessary to clarify this point, because only a relatively small number of participants answered about their lipid profile at home, the ages of participants who answered varied greatly, and the times following injury or following discharge from hospital were also inconsistent.

Further studies would evaluate about other factors also contributing to CHD risk (such as diabetes mellitus, smoking, homocysteine, uric acid, ferritin, and creatinine).

\section{CONCLUSIONS}

In a Japan-based study, it was found that in individuals with high tetraplegia (above C4), TC, HDL-C, and LDL-C were significantly lower than in healthy controls, a finding consistent with previous studies conducted in tetraplegia below $\mathrm{C} 5$ and paraplegia. The TC/HDL-C and LDL-C/HDL-C ratios of high tetraplegia were within normal range. To conclude the incidence of CHD high tetraplegia must be evaluated with a larger sample and other variables.

\section{REFERENCES}

[1] Sodan RJ. Causes of death after spinal cord injury. Spinal Cord 2000; 38: 604-10.

[2] Bauman WA, Spungen AM, Zhong Y, et al. Depressed serum high density lipoprotein cholesterol levels in veterans with spinal cord injury. Paraplegia 1992; 30: 697-703.

[3] Maki KC, Briones ER, Langbein WE, et al. Associations between serum lipids and indicators of adiposity in men with spinal cord njury. Paraplegia 1995; 33: 102-9.

[4] Bauman WA, Adkins RH, Spungen AM, et al. The effect of residual neurological deficit on serum lipoproteins in individuals with chronic spinal cord injury. Spinal Cord 1998; 36: 13-7.

[5] Walker J, Shephard RJ. Cardiac risk factors immediately following spinal injury. Arch Phys Med Rehabil 1993; 74: 1129-33.

[6] Garshick E, Kelley A, Cohen SA, et al. Prospective assessment of mortality in chronic spinal cord injury. Spinal Cord 2005; 43: 408-16.

[7] El-Sayed MS, Younesian A. Lipid profiles are influenced by arm cranking exercise and training in individuals with spinal cord injury. Spinal Cord 2005; 43(5): 299-305.

[8] Friedewald WT, Levy RI, Fredrickson DS. Estimation of the concentration of low-density lipoprotein cholesterol in plasma without the use of a preparative ultracentrifuge. Clin Chem 1972; 18: 499-502.

[9] Ryousuke I. Incomplete Quadriplegia-incidence,cause of injuries, outcome. Sogo Rehabil 2000; 28: 317-21.

[10] Japan Atherosclerosis Society(JAS). Guidelines for Diagnosis and Treatment of Atherosclerotic Cardiovascular Diseases. MDS Co. 2002.

[11] Krum H, Howes LG, Brown DJ, et al. Risk factors for cardiovascular disease in chronic spinal cord injury patients. Paraplegia 1992; 30: 381-8.

[12] Brenes G, Dearwater S, Shapera R, et al. High density lipoprotein cholesterol concentrations in physically active and sedentary spinal cord injured patients. Arch Phys Med Rehabil 1986; 67: 445-50.

[13] Dallmeijer AJ, Hopman MTE, van der Woude LHV. Lipid, lipoprotein, and apolipoprotein profiles in active and sedentary men with tetraplegia. Arch Phys Med Rehabil 1997; 78: 1173-6.

[14] Choi S, Lee SH, Lee JY, et al. Factors affecting prognosis of patients who underwent corpectomy and fusion for treatment of cervical ossification of the posterior longitudinal ligament: analysis of 47 patients. J Spinal Disord Tech 2005; 18: 309-14.

[15] Stampfer MJ, Sacks FM, Salvini S, et al. A prospective study of cholesterol, apolipoproteins, and the risk of myocardial infarction. New Engl J Med 1991; 325: 373-81

[16] Apstein MD, George BC. Serum lipids during the first year following acute spinal cord injury. Metabolism 1998; 47: 367-70. 